\title{
Wavelet Correlation Transform Method and Gradient Method to Determine Aerosol Layering from Lidar Returns: Some Comments
}

\author{
ADOLFO COMERÓN \\ RSLab, Universitat Politècnica de Catalunya, Barcelona, Spain \\ Michä̈L SiCARD AND FRANCESC ROCADENBOSCH \\ RSLab, and IEEC-CRAE/UPC, Universitat Politècnica de Catalunya, Barcelona, Spain
}

(Manuscript received 26 October 2012, in final form 4 January 2013)

\begin{abstract}
Identification of aerosol layers on lidar measurements is of interest to determine ranges where aerosol properties are likely to be homogeneous and to infer transport phenomena and atmosphere dynamics. For instance, the range-corrected backscattered signal from aerosol measured with lidars has long been used as a proxy to determine the depth of the planetary boundary layer. The method relies on the assumption that in a well-mixed atmosphere, a rather homogenous aerosol distribution will exist within the boundary layer; hence, a sudden drop in the lidar range-corrected signal profile will mark the end of the layer. The most usual methods to detect that drop are the gradient method, which detects a negative maximum in the derivative with respect to range of the lidar range-corrected signal, or of its logarithm, and the wavelet correlation transform method, which detects a maximum in the correlation function of the lidar range-corrected signal and a wavelet, usually the Haar wavelet. These methods are not restricted to determining the boundary layer height but can also be used to locate the edges of lofted aerosol layers. Using fundamentals of linear system theory, this study shows the deep link existing between the gradient method and the wavelet correlation transform method using the Haar wavelet, the latter being equivalent to the gradient method applied to a range-corrected signal profile smoothed by a low-pass spatial filtering, which seems not to have been explicitly noted in the literature so far. Consequences are readily drawn for the wavelet correlation transform method using other wavelets.
\end{abstract}

\section{Introduction}

The range-resolved backscatter signal of elastic lidars contains information from which the height of aerosol layers can be derived. Identification of these layers is important in atmospheric observations to determine ranges where aerosol properties are likely to be homogeneous, as well as to infer transport phenomena and atmosphere dynamics. A conspicuous example of the latter application is the use of lidar backscatter profiles to measure the planetary boundary layer depth.

The planetary boundary layer (PBL) can be defined as the lowest part of the troposphere that is directly influenced by the ground and whose changes are shown in less than $1 \mathrm{~h}$ (Stull 1988). Factors related to the orography,

Corresponding author address: Adolfo Comerón, Universitat Politècnica de Catalunya, c/ Jordi Girona, 1-3, Edif. D3-202, E-08034 Barcelona, Spain.

E-mail: comeron@tsc.upc.edu the season, the daytime, or the weather act over the PBL and change its structure both spatially and temporally. Its vertical extent, called PBL depth or PBL height, varies from a few tens of meters to several kilometers. The PBL depth is a meteorological parameter with a strong influence on pollutant dispersion behavior and, eventually, on living organisms' health. Emitted pollutants tend to be trapped inside the boundary layer in such a manner that for a given emission rate, low depths of the PBL will tend to increase the concentration of particles and gases close to the ground (Pérez et al. 2004). The PBL depth is therefore an important input parameter for numerical weather and air quality prediction models.

The aerosol distribution as measured by lidars has long been used as a proxy to determine the PBL depth. Aerosols are also trapped inside the PBL. Although the aerosol profile cannot unambiguously tell the extent of the boundary layer-mostly because of the aerosol mixing inside the PBL and/or possible layers above the PBL (Sicard et al. 2006) — in many instances a sudden 
drop in a range-corrected lidar profile will be related to the PBL height. Although not in a straightforward manner in general, peaks of the negative derivative of the range-corrected lidar profile or of its logarithm, subject to certain constraints, have been successfully used to detect the depth of the boundary layer (Senff et al. 1996; Hayden et al. 1997; Flamant et al. 1997); the effectiveness of combining the information given by the first and second derivatives of the backscatter rangecorrected profile, subject also to certain conditions, has been demonstrated by Sicard et al. (2006). Those methods are called gradient methods. Likewise, the socalled wavelet correlation-or covariance-transform (WCT) methods (Cohn and Angevine 2000; Brooks 2003; Morille et al. 2007; Baars et al. 2008) have also been used to track layering features in the range-corrected lidar profile. In the WCT method the lidar profile is correlated with a function of a specifically chosen form, the so-called wavelet. The shift of the wavelet with respect to the lidar profile for which a maximum of the correlation is obtained identifies, under certain constraints and subject to some uncertainty, as for the gradient methods, the position of the PBL height or, for that matter, the positions of layer edges and/or peaks. A variable dilation parameter (scale) in the wavelet has also been used to try to detect the peaks corresponding best to the PBL height, with the aim of achieving robust methods able to process large amounts of data in an automated way. Brooks (2003) used also the WCT technique to determine the limits of the transition zone and studied empirically the effect of the wavelet dilation on the spatial spectrum of the WCT. As compared to gradient methods, which use numerical differentiation prone to be adversely affected by noise, WCT methods use instead a correlation integral, which, in addition, can be very efficiently calculated by standard numerical computation packages.

Although most of the reported applications of gradient and WCT methods deal with the determination of the PBL depth, the methods can obviously also be applied to determine the range of other layers [see, e.g., Morille et al. (2007) with respect to WCT].

In the following sections we show the close link existing between gradient methods and WCT methods, and, more specifically, that the WCT method using the Haar wavelet is in fact equivalent to the gradient method applied to a signal smoothed by a low-pass spatial filtering.

\section{The gradient method and the WCT method}

The range-corrected lidar signal is denoted by $s(x)$. To detect steep decreasing gradients in the gradient method, either $-[d s(x) / d x]$ or $-[d \ln s(x) / d x]$ is computed. Falling steps on $s(x)$ are identified by the positions of the relative

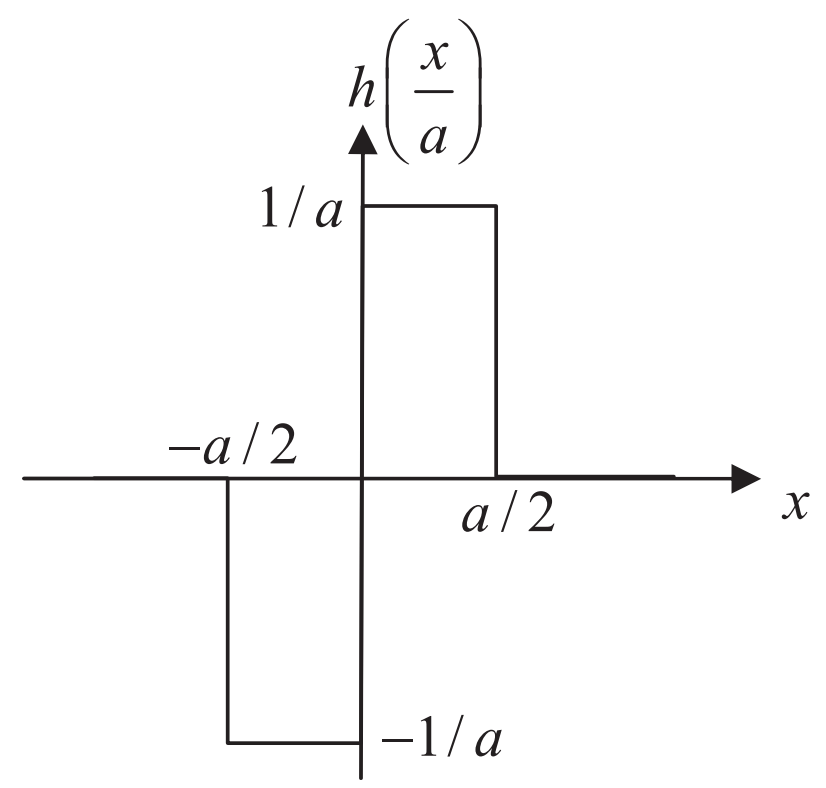

FIG. 1. Definition of the Haar wavelet.

maxima of these derivatives; likewise, derivative relative minima mark the position of increasing steps on $s(x)$. Concerning the PBL, one of the first peaks of the derivative profile corresponds approximately to the position of the PBL top if some physical-related constraints are met (Sicard et al. 2006). Different maxima and minima can also be used to identify the edges of different aerosol layers. Because the differentiation operation tends to enhance high frequencies, the method can be impaired by noise corrupting the signal.

In the WCT method, the range-corrected signal $s(x)$ is convolved with a wavelet $w(x)$ within a given range $\left(x_{\min }, x_{\max }\right)$ of validity of $s(x)$, outside of which $s(x)$ can formally considered to be 0 :

$$
c(x)=\int_{x_{\min }}^{x_{\max }} s\left(x^{\prime}\right) w\left(x-x^{\prime}\right) d x^{\prime} .
$$

The position of the maxima and minima of $c(x)$ marks the edges of aerosol layers, one of which, subject to physical or empirical constraints, may correspond to the PBL top.

\section{The WCT method with the Haar wavelet}

A frequently used form of $w(x)$ is the so-called Haar wavelet (see Fig. 1), which can be defined as

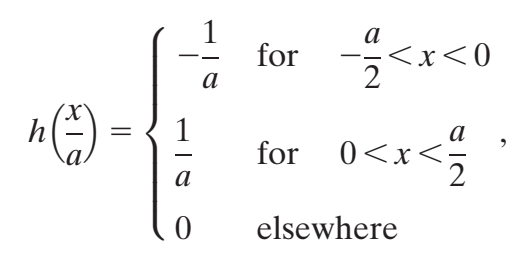




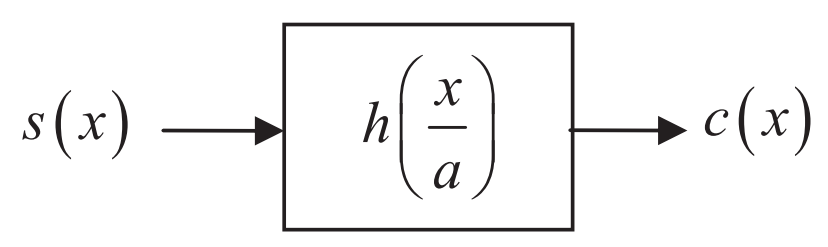

FIG. 2. The Haar WCT as the output of a linear system with impulse response $h(x / a)$ and input $s(x)$.

where $a$ is called the wavelet dilation. From the point of view of linear system theory, $c(x)$ defined by Eq. (1) can be considered as the output of a filter with impulse response $w(x)=h(x / a)$ when the input is $s(x)$ (Fig. 2).

To gain insight into the operation performed by the filter of Fig. 2, we note that

$$
h\left(\frac{x}{a}\right)=-\frac{d}{d x} \Lambda\left(\frac{x}{a}\right)
$$

with

$$
\Lambda\left(\frac{x}{a}\right)= \begin{cases}\frac{1}{a}\left(\frac{a}{2}+x\right) & \text { for } \quad-\frac{a}{2}<x<0 \\ \frac{1}{a}\left(\frac{a}{2}-x\right) & \text { for } 0<x<\frac{a}{2} \\ 0 & \text { elsewhere }\end{cases}
$$

(see also Fig. 3). Therefore, we can consider the filter of Fig. 2 as the cascade of a filter with impulse response $\Lambda(x / a)$ and an ideal differentiation filter (Fig. 4). But a filter with impulse response $\Lambda(x / a)$ as given by Eq. (4) is a low-pass filter with transfer function

$$
H_{\Lambda}(f)=\int_{-\infty}^{\infty} \Lambda\left(\frac{x}{a}\right) e^{-j 2 \pi f x} d x=\frac{a}{4} \operatorname{sinc}^{2}\left(\frac{a f}{2}\right),
$$

(Fig. 5), where $f$ is the spatial frequency and $\operatorname{sinc}(t)=$ $\sin (\pi t) / \pi t$.

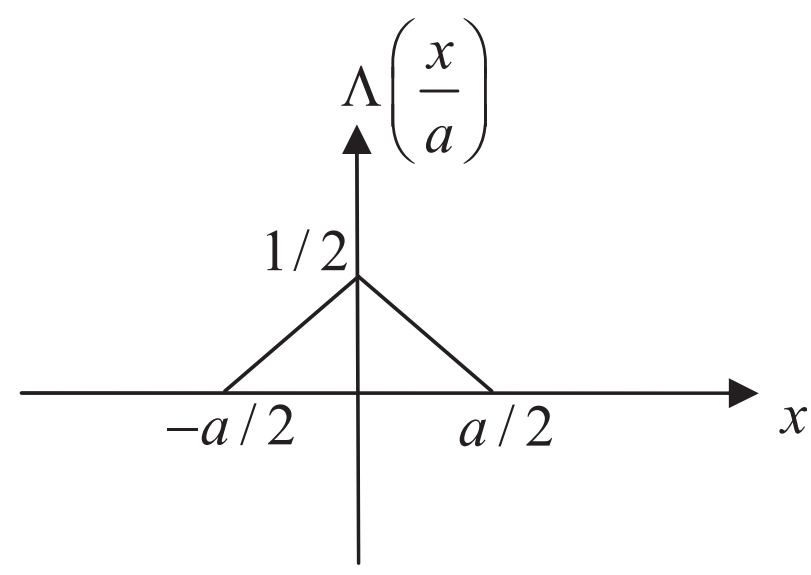

FIG. 3. Definition of the $\Lambda(x / a)$ function.

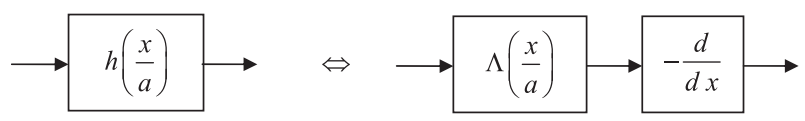

FIG. 4. The filter of Fig. 2 as the cascade of a filter with impulse response $\Lambda(x / a)$ followed by an ideal differentiator.

From the above considerations, it turns out that computing the wavelet correlation transform with the Haar wavelet is equivalent to filtering the range-corrected signal in the spatial frequency domain with a low-pass filter of bandwidth approximately $1 / a$, and differentiating the filter output; in this sense, the WCT method with the Haar wavelet can be considered as a particular form of the gradient method.

Figure 6 shows with an example using real digitized signals the virtual identity of both approaches, that is, on the one hand smoothing $s(x)$ by filtering it with a filter of impulse response like that of Fig. 3 and differentiating the filtering result and, on the other hand, convolving $s(x)$ with the Haar wavelet. In this example, the rangecorrected signal corresponds to a 532-nm elastic channel of a lidar with a frequency-doubled Nd:YAG laser and an acquisition system sampling the return signal every $3.75 \mathrm{~m}$. The rising slope in the ranges close to $0 \mathrm{~m}$ is due to incomplete overlap effects. The dilation parameter $a$ is taken to be $200 \mathrm{~m}$.

\section{Conclusions}

It has been shown that the WCT method using a Haar wavelet is completely equivalent to the gradient method applied to a spatially low-pass filtered range-corrected signal. Because of the linearity of the filter and derivative

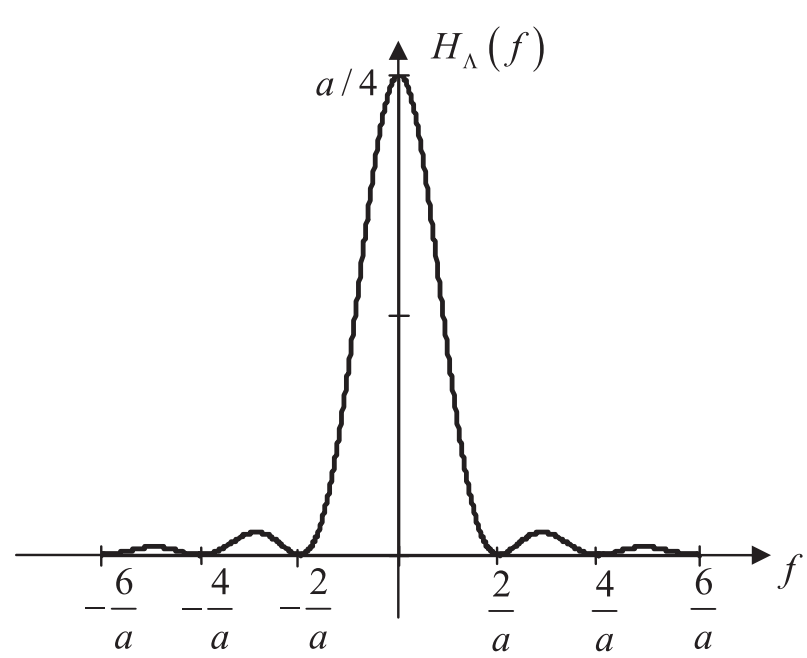

FIG. 5. Transfer function corresponding to a filter with impulse response $\Lambda(x / a)$. The letter $f$ denotes the spatial frequency. 


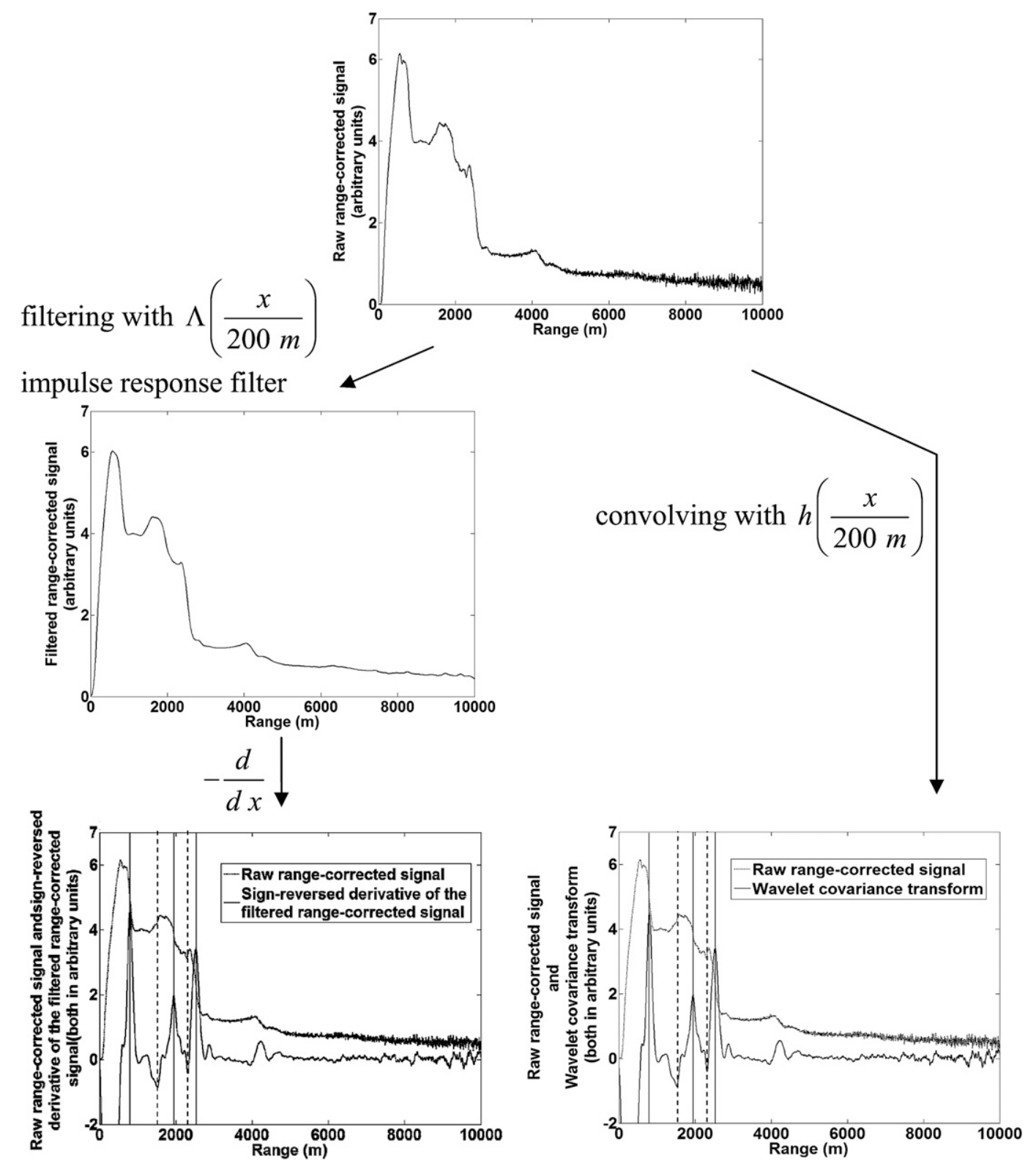

FIG. 6. Practical illustration of the equivalence between the determination of slope maxima in the range-corrected signal (top) by computation of the WCT with the Haar wavelet (right arrow) and by computing the reverse-sign derivative of the range-corrected signal filtered with a $\Lambda(x / 200 \mathrm{~m})$ impulse response filter (left arrow). The positive peaks in both the derivative and the WCT mark relative maxima (continuous vertical lines) on the reversed-sign slope of the range-corrected signal, approximately coincident with the end of layers; the negative peaks (dashed vertical lines) mark relative minima on the reversed-sign slope of the range-corrected signal, corresponding approximately to the start of layers. If the curves of the left and right bottom panels were superimposed on the same graph, they would be virtually indistinguishable.

operators, the same result would also be obtained if the range-corrected signal is first differentiated and the result is filtered with the same low-pass filter.

The generalization of these results to other wavelet forms is immediate. For example, if the wavelet has an odd symmetry, it can be considered as the derivative of an even function. As long as this function corresponds to the impulse response of a low-pass filter, which it will in many practical cases, the WCT method can be considered as a gradient method applied to a low-pass filtered version of the range-corrected signal-the exact details of the filtering depending on the wavelet form. 
As another example, consider the "Mexican hat" wavelet used by Morille et al. (2007), which is the second derivative of a Gaussian function. Following a reasoning parallel to that developed in section 3, one concludes that using this wavelet is equivalent to smoothing the range-corrected signal with a low-pass filter with Gaussian impulse response and differentiating twice the filter output, which is consistent with the claimed ability of this wavelet to detect the layer base, top, and peak.

In spite of the equivalence of WCT and filtering-anddifferentiation (or differentiation-and-filtering) methods, it is clear that WCT presents the advantage of performing all the operations in a single, computationally efficient step.

Acknowledgments. This work is supported by the European Union Seventh Framework Programme (FP7 2007-2013) project Aerosols, Clouds, and Trace Gases Research Infrastructure Network (ACTRIS) (Grant Agreement 262254); and by the Spanish Ministry of Science and Innovation and FEDER funds under the Projects TEC2009-09106/TEC and CGL2011-13580-E/CLI.

\section{REFERENCES}

Baars, H., A. Ansmann, R. Engelmann, and D. Althausen, 2008: Continuous monitoring of the boundary-layer top with lidar. Atmos. Chem. Phys., 8, 7281-7296.
Brooks, I. M., 2003: Finding boundary layer top: Application of a wavelet covariance transform to lidar backscatter profiles. J. Atmos. Oceanic Technol., 20, 1092-1105.

Cohn, S., and W. M. Angevine, 2000: Boundary layer height and entrainment zone thickness measured by lidars and windprofiling radars. J. Appl. Meteor., 39, 1233-1247.

Flamant, C., J. Pelon, P. H. Flamant, and P. Durand, 1997: Lidar determination of the entrainment zone thickness at the top of the unstable marine atmospheric boundary layer. Bound.Layer Meteor., 83, 247-284.

Hayden, K. L., and Coauthors, 1997: The vertical chemical and meteorological structure of the boundary layer in the Lower Fraser Valley during Pacific '93. Atmos. Environ., 31, 2089-2105.

Morille, Y., M. Haeffelin, P. Drobinski, and J. Pelon, 2007: STRAT: An automated algorithm to retrieve the vertical structure of the atmosphere from single-channel lidar data. J. Atmos. Oceanic Technol., 24, 761-775.

Pérez, C., M. Sicard, O. Jorba, A. Comerón, and J. M. Baldasano, 2004: Summertime re-circulations of air pollutants over the northeastern Iberian coast observed from systematic EARLINET lidar measurements in Barcelona. Atmos. Environ., 38, 3983-4000.

Senff, C., J. Bösenberg, G. Peters, and T. Schaberl, 1996: Remote sensing of turbulent ozone fluxes and the ozone budget in the convective boundary layer with DIAL and Radar-RASS: A case study. Contrib. Atmos. Phys., 69, 161-176.

Sicard, M., C. Pérez, F. Rocadenbosch, J. M. Baldasano, and D. García-Vizcaino, 2006: Mixed-layer depth determination in the Barcelona coastal area from regular lidar measurements: Methods, results and limitations. Bound.-Layer Meteor., 119, 135-157.

Stull, R. B., 1988: An Introduction to Boundary Layer Meteorology. Atmospheric and Oceanographic Sciences Library, Vol. 13, Kluwer Academic Publishers, 670 pp. 